\title{
Treadmill exercise improves memory and increases hippocampal BDNF in a rat model of Alzheimer's Disease
}

\author{
Rokhsareh Abshenas ${ }^{1,2}$, Tayebe Artimani ${ }^{1,2}$, Iraj Amiri ${ }^{1,2}$, Siamak Shahidi $^{3}$, Sara Soleimani Asl ${ }^{1,2 *}$ iD \\ 1. Endometrium and Endometriosis Research Centre, Hamadan University of Medical Sciences, Hamadan, Iran \\ 2. Anatomy Department, School of Medicine, Hamadan University of Medical Sciences, Hamadan, Iran \\ 3. Physiology Department, School of Medicine, Hamadan University of Medical Sciences, Hamadan, Iran
}

\begin{abstract}
Introduction: Alzheimer's disease is strongly correlated with learning and memory impairments. As exercise can enhance memory and learning, in this study, we have investigated the effects of treadmill exercise on memory impairment in amyloid $\beta$ $(A \beta)$ - treated rats focusing on brain-derived neurotrophic factor (BDNF) expression.

Methods: Wistar male rats received intracerebroventricular (ICV) injection of $A \beta$ and exercised on a treadmill for one month. Memory function was assessed using Morris water maze (MWM) and avoidance learning tasks. The level of BDNF was examined by the ELISA test.
\end{abstract}

Results: The results of MWM and avoidance learning tasks showed that treadmill exercise could improve $A \beta$ - induced memory impairment significantly. Moreover, BDNF expression increased following exercise in the $A \beta$ - treated rats.

Conclusion: The present results suggested that treadmill exercise may improve memory in Alzheimer's disease by increasing BDNF level in the hippocampus.

\section{Keywords:}

Treadmill exercise;

Alzheimer's disease;

Memory;

BDNF

\section{* Corresponding author:}

S. Soleimani Asl

Email:

s.soleimaniasl@umsha.ac.ir

Tel: +98 (81) 38380208

Received 10 November 2019;

Received in revised form 11 June 2020; Accepted 15 June 2020

http://dx.doi.org/10.32598/ppj.24.4.50

\section{Introduction}

Alzheimer's disease (AD) the most common cognitive disorders of the elderly is characterized by senile plaques of amyloid $\beta(A \beta)$ and neurofibrillary tangles (Selkoe, 1997). The deposition of fibrillary $A \beta$ is strongly accepted as an agent in the pathogenesis of $A D$ and associated with reduced cognition, increased reactive oxygen species and decreased cell count in the hippocampus (De Felice et al., 2007). It has been reported that up-regulation of BDNF leads to a decrease in $A \beta$ - induced neurotoxicity (Doi et al.,
2013). Cognition and memory functions are positively affected by brain-derived neurotrophic factor (BDNF) (Kim and Kim, 2013). BDNF is a member of the neurotrophin family of growth factors, which has been expressed in the highest level in the brain especially in the hippocampus and facilitates the release of glutamate and enhances the phosphorylation of the NR1 and NR2B subunits of the NMDA-receptor complex (Tyler and Pozzo-Miller, 2001). BDNF pathway is mediated by TrkB and p75 receptors that trigger the phosphatidyl-inositol 3 kinase, 
phospholipase C gamma and intracellular signalregulated kinase 1/2 signaling cascades (for more detail see a review by Bekinschtein et al.) (Bekinschtein et al., 2008).

Treadmill exercise has been accepted as a therapeutic strategy that induces protection in the brain of both human and rodents (Gharebaghi et al., 2017; Otsuka et al., 2016). Several studies suggest that exercise improves learning and memory function through an increase in the levels of BDNF (Jeon and $\mathrm{Ha}, 2017)$. Furthermore, treadmill exercise enhances neurogenesis and myelin repairment via the $\mathrm{Wnt} 3 / \beta$ catenin signaling pathway and induces an increase in the expression of BDNF and myelin basic protein (Cheng et al., 2020) .

In the present study, we hypothesized that treadmill exercise might improve $A \beta$ - induced memory impairment through an increase in BDNF in the hippocampus of rats.

\section{Materials and methods}

\section{Animals}

Thirty-five adult male Wistar rats (250-300g) were obtained from the animal facility of Hamadan University of Medical Sciences (HUMS) and maintained under standard laboratory condition (12 $\mathrm{h} / \mathrm{h}$ light/dark cycle, $20 \pm 2^{\circ} \mathrm{C}$ and $50 \%$ relative humidity) with free access to food and water. All experiments approved by the Ethical committee of HUMS (No: IR.UMSHA.AC.REC.1396.99). The rats randomly classified into four groups $(n=7$ per each group): control, sham-operated, $A \beta$ and $A \beta+$ exercise groups.

\section{Injection of $\mathrm{A} \beta$}

To induce $A D$, we performed intracerebroventricular (ICV) injection of $A \beta$ (1-42, Sigma-Aldrich, St Louis, MO, USA) according to the previously described method (Komaki et al., 2019). Briefly, anesthetized rats were placed in a stereotaxic frame and the skull drilled over the lateral ventricle using the following coordinate: AP: $-0.9 \mathrm{~mm}$ from the bregma; ML: $1.6 \mathrm{~mm}$ from the midline; DV: $2.0 \mathrm{~mm}$ from the skull surface (Paxinos and Watson, 2006) and $A \beta(5 \mu \mathrm{g} / 5 \mu \mathrm{l})$ was injected slowly. The shamoperated group went under surgery similarly to the $A \beta$ group except for $A \beta$ injection.

$A \beta$ peptides (1-42) represent very different conformational states so that the residues $31-34$ and 38-41form a $\beta$-hairpin, which causes a reduction in the flexibility of $\mathrm{C}$-terminal and the greater propensity of $A \beta 42$ to form amyloids (Chen et al., 2017).

\section{Treadmill exercise protocol}

The day after the injection of $A \beta$, we used a motorized rodent treadmill instrument (Tajhiz Gostare Omide Iranian, Iran) to exercise according to a previously published protocol (Gharebaghi et al., 2017). The rats ran on the treadmill for one month (30min daily and five constitutive days/week). The exercise consisted of running at the speed of $25 \mathrm{~m} / \mathrm{min}$ with a $0.3 \mathrm{~mA}$ stimulus current electric shock when the rats entered the rear of the test chamber.

\section{Morris water maze (MWM) task}

spatial memory was assessed (Gharebaghi et al., 2017) using water filled MWM $(210 \times 51 \mathrm{~cm})$, the day after the last day of ran on the last day of ran on the treadmill. There was a hidden platform located at a fixed position in the pool. Four consecutive training days consisting of two-block with four trials were conducted. In each trial, the rats were allowed to swim in the pool for $60 \mathrm{~min}$ at different points. There were 30 s and $5 \mathrm{~min}$ inter-trial and inter-block intervals, respectively. Escape latency and the distance to reach (traveled distance) the hidden platform were recorded as the parameter of acquisition memory Using a camera located above the center of the maze. On day 5, the platform was removed and the percentage of time spent in the target quadrant assessed as a parameter of the retention of memory.

\section{Inhibitory avoidance apparatus (Shuttle-box)}

The shuttle box apparatus consisted of two compartments (white and black) separated with a guillotine door. There was a stainless steel shock grid floor in the dark compartment. The day after the spatial memory assessment by MWM, the rats were placed in the white chamber and after $5 \mathrm{~s}$ the guillotine door was opened and the rats entered the dark compartment. The door was closed and the rats received an electronic foot shock $(50 \mathrm{~Hz}$ and $1.5 \mathrm{~mA}$ intensity) for $3 \mathrm{~s}$. After $24 \mathrm{~h}$, the time in the dark compartment (TDC) and step through latency (STL) were recorded to evaluate the avoidance learning memory. The latency was recorded a maximum of 300 s. 

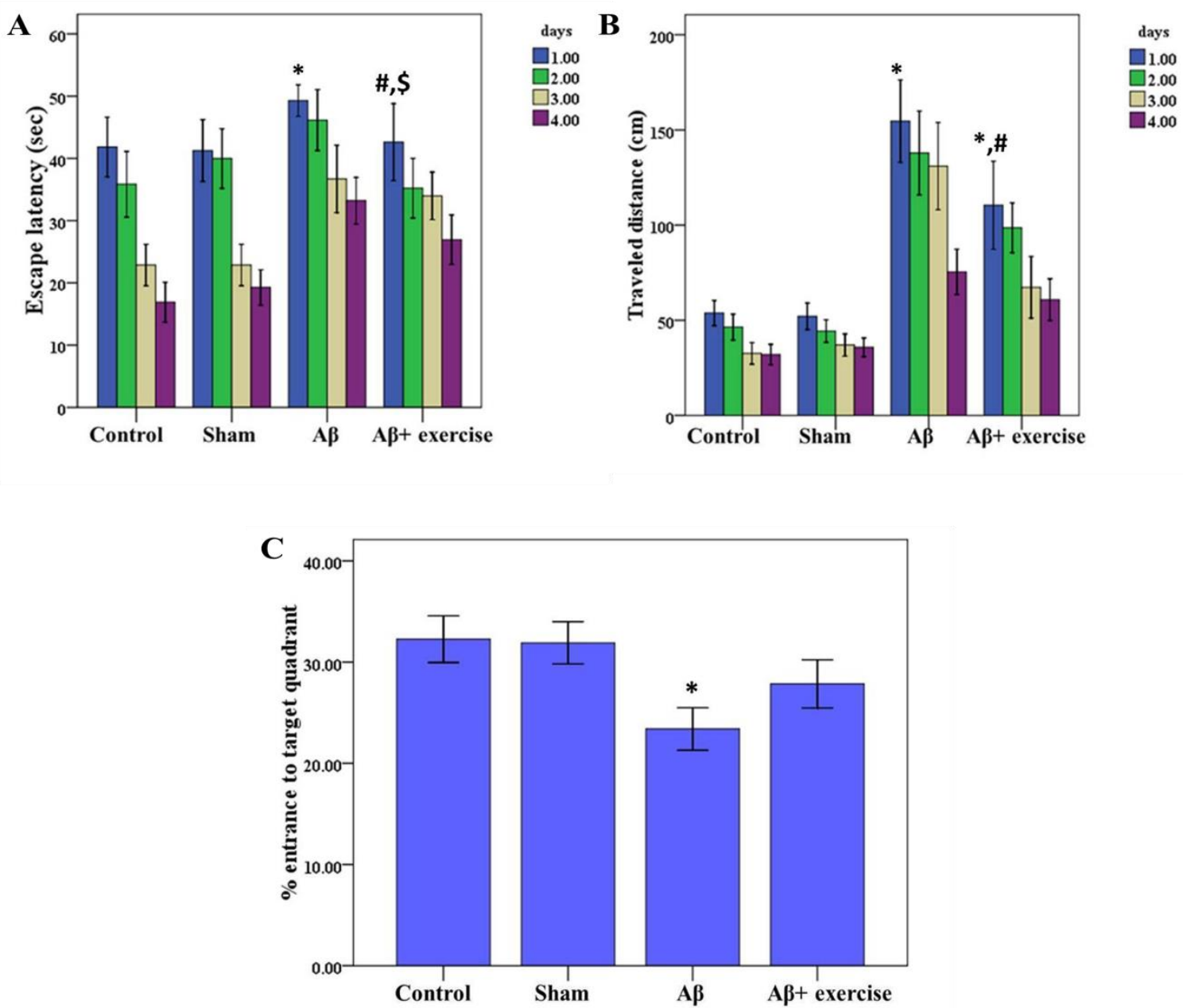

Fig.1. Protective effects of treadmill exercise in $A \beta$ (1-42)-induced Alzheimer's model in the water-maze test. Each block signifies the mean of latencies $(A)$ and traveled distance $(B)$ to reach the hidden platform during four consecutive trial days in the MWM. A: ${ }^{\star} P<0.001$ vs. control and sham groups; ${ }^{\#} \mathrm{P}<0.05$ vs. control and sham groups; ${ }^{\$} P<0.01$ vs. $A \beta$ group. $B$ : ${ }^{*} P<0.001$ vs. control and sham groups; ${ }^{\#} P<0.001$ vs. A $\beta$ group. C: Represents the mean of the percentage of the entrance to the target quarter in the probe trial in the MWM, ${ }^{\star} P<0.05$ vs. control group. Each value is the mean \pm SEM.

\section{Enzyme-linked immunosorbent assay (ELISA)}

Three hippocampi from each group were mixed and sonicated in PBS. BDNF level was quantified using an ELISA kit (ZellBio, Ulm, Germany) according to the manufactures recommendation. Assays were carried out in duplicate trials.

\section{Statistical analysis}

Statistical analysis was performed using SPSS 16. The repeated measure, one- way analysis of variance (ANOVA) and Tukey's multiple comparison tests were used to analyze the significance between the groups. $P$-value $<0.05$ was considered significant.

\section{Results}

\section{MWM performance}

To assess acquisition memory, escape latency and traveled distance of four constitutive days were analyzed using two-way ANOVA test with treatment as one factor and training days as the second factor. The results of escape latency showed a significant effect for training days $[F(3,2049)=81.85, P<0.001]$ and treatment $[F(4,44.03)=15.52, P<0.001]$. There was a significant interaction between training days and treatment $[F(12,538)=2.14, P<0.05]$. Further analysis indicated that $A \beta$ - treated rats took more time to reach the hidden platform than control and sham-operated groups $(P<0.001$, Fig. $1 \mathrm{~A})$. According to the results, treadmill exercise for one month caused a significant reduction in escape latency in comparison to the $A \beta$ group $(P<0.01)$. The results of traveled distance showed a significant effect for training days $[F(3,5890)=53.68, \quad P<0.001]$ and treatment $[F(4,3032)=276.4, P<0.001]$. There was a significant interaction between treatment and training days $[F(12,7844)=7.15, P<0.001]$. One-way ANOVA 

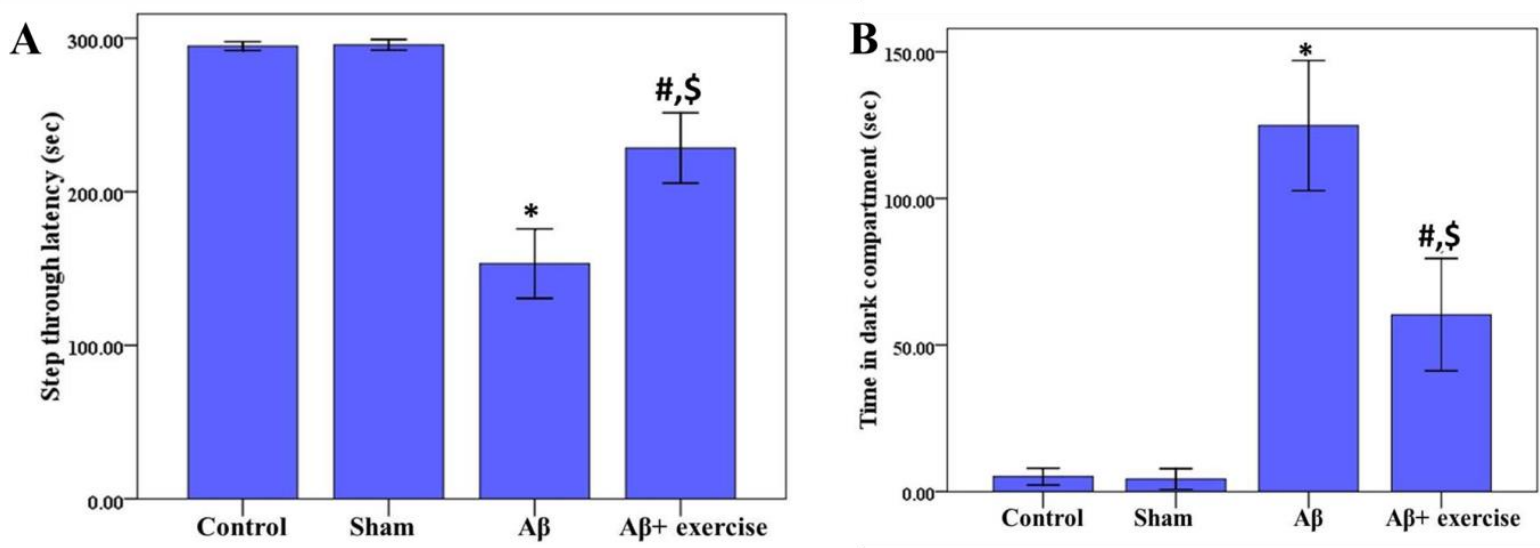

Fig.2. Protective effects of treadmill exercise in $A \beta$ (1-42)-induced Alzheimer's model in the Shuttle box apparatus. The mean of step-through latency $(A)$ and time spent in the dark compartment $(B)$ in the passive avoidance task. $A:{ }^{*} P<0.001$ vs. control and sham groups; ${ }^{\#} P<0.05$ vs. control and sham groups; ${ }^{\$} P<0.05$ vs. A $\beta$ group. $B:{ }^{*} P<0.001$ vs. control and sham groups; ${ }^{\#}<<0.05$ vs. control and sham groups; ${ }^{\$} P<0.05$ vs. A $\beta$ group. Each value is the mean \pm SEM.

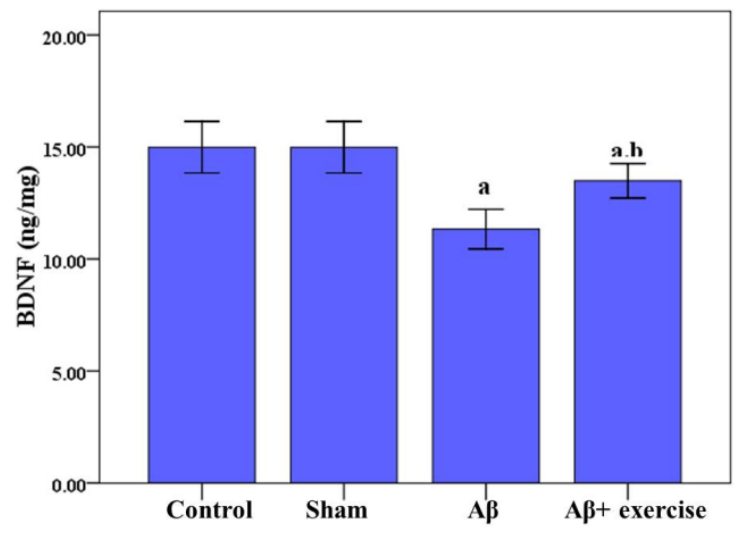

Fig.3. The effects of $A \beta$ and treadmill exercise on the BDNF concentration of hippocampus homogenate samples. Mean value $\left( \pm\right.$ SEM) of BDNF level in the hippocampus. ${ }^{\star} P<0.001$ vs. control and sham groups; ${ }^{\#} P<0.05$ vs. $A \beta$ group.

analysis of training days revealed that $A \beta$-treated group swam further to reach the platform, which was significant when compared to the control and shamoperated groups $(P<0.001$, Fig. 1B). Treadmill exercise decreased traveled distance compared with $\mathrm{A} \beta$ group $(P<0.001)$.

In the probe trial session, we found a significant difference between $A \beta$-treated rats and the control group ( $P<0.05$, Fig. $1 C$ ) and $A \beta$ group spent less time in the target quadrant. Treadmill exercise caused an increase in the entrance to the target quadrant $(27.85 \pm 2.37)$ compared with A -treated rats $(23.39 \pm 2.10)$ without any significant difference.

\section{Passive avoidance task}

Our results revealed a significant difference between control, sham and $A \beta$ groups $(P<0.001$, Fig. $2 A)$ in STL. $A \beta$-treated rats that exercised for one month showed a significant increase in STL concerning the
$A \beta$ group $(P<0.01)$. Furthermore, control and shamoperated groups spent less time in the dark compartment compared to the $A \beta$ group $(P<0.001$, Fig. 2B). Treadmill exercise significantly attenuated TDC when compared to the $A \beta$ group $(P<0.01)$.

\section{ELISA for BDNF}

As shown in Figure 3, the ICV injection of $A \beta$ led to a significant reduction in the BDNF level in the hippocampus compared with the control and sham group $(P<0.001)$. We found a significant increase in BDNF level in rats undergoing exercise than $A \beta$ treated rats $(P<0.05)$.

\section{Discussion}

Learning and memory impairments are the important symptoms of $A D$ and ICV injection of $A \beta$ resulted in the structural and physiological alterations in the hippocampus, which contribute to cognitive deficit 
(Leuner et al., 2012; van der Zee et al., 2008). In this study, we found an attenuation of learning and memory impairment by treadmill exercise in $A \beta$ induced AD model. Second, we showed that exercise could improve impaired memory through increased expression of BDNF in the hippocampus.

Previous studies have found a robust relation between physical exercise and cognition. They reported that exercise increases life span and prevents the decline of behavioral performance in middle age (Navarro et al., 2004) and the elderly (Kim et al., 2010). Treadmill exercise increased latency of the step-down avoidance and also decreased the latency and distance in MWM in the $A \beta$-injected rats, indicating that exercise attenuates $A \beta$ - induced memory impairment. Our results supported finding by Kim et al. (2010) who reported that treadmill exercise is a useful strategy for preventing failure of memory in the elderly.

Physical exercise has also been shown to enhance cognitive function in a rat model of vascular dementia (Choi et al., 2016) and Alzheimer's disease (Koo et al., 2017). They concluded that treadmill exercise improves cognition deficits, possibly by increasing disintegrin and metalloproteinase domain-containing protein 10 . In another study, latency in the passive avoidance test increased in MPTP (1-methyl-4phenyl-1,2,3,6-tetrahydropyridine)/probenecid-

induced Parkinson's disease in the mice model undergoing exercise (Sung, 2015). They reported an increased BDNF expression in the ventral midbrain of exercised animals and suggested that exercise can overcome Parkinson- induced memory impairment through enhancement of BDNF expression and prevention of dopaminergic neuronal damage.

BDNF as a member of the neurotrophic factors family plays an important role in cognition and memory function, neuronal survival and differentiation (Pang and $\mathrm{Lu}, 2004)$. It has been reported that exogenous application of BDNF improved hippocampal long-term potentiation impairment in BDNF knockout mice (Patterson et al., 1996). In the present study, the ICV injection of $A \beta$ caused a reduction in the expression of BDNF in the hippocampus and a significant increase observed in BDNF expression in rats that underwent treadmill exercise. Consistent with our results, Molteni et al. (2002) suggested that exercise increased the expression of plasticity-related genes such as BDNF in the rat hippocampus.
In another study, exercise induced the synaptic plasticity markers through a BDNF- mediated mechanism in the hippocampus (Vaynman et al., 2003). Previously we found that treadmill exercise for one month alleviated cognitive deficit most likely by an increase in the BDNF expression (Sajadi et al., 2017). BDNF regulates neurogenesis, axonal and dendritic branching, and remodeling, as well as functional maturation of excitatory and inhibitory synapse (Seil and Drake-Baumann, 2000; VicarioAbejón et al., 1998). Several studies have established a positive correlation between BDNF expression and the memory function (Slipczuk et al., 2009). They showed over-expression of BDNF increased neurogenesis in the hippocampus and improved spatial memory (Rossi et al., 2006). We found a significant increase in BDNF expression following exercise that involved long-term plasticity and memory. Based on the combined findings of this study, it can be inferred that treadmill exercise protects against memory impairment in $A D$ model through an increase in BDNF expression.

\section{Conclusion}

In this study, avoidance learning and spatial memory in the $A \beta$-injected rats that underwent exercise were much better than those in the AD model. Our study revealed that BDNF expression was enhanced following exercise in the hippocampus. Therefore, it was concluded that treadmill exercise could be an important clinical strategy for preventing failure of memory in those with neurodegenerative disease.

\section{Acknowledgments}

This study was supported by the Hamadan University of Medical Sciences grant (9603201344).

\section{Conflict of interest}

The authors report no conflict of interest.

\section{References}

Bekinschtein P, Cammarota M, Izquierdo I, Medina JH. BDNF and memory formation and storage. Neuroscientist 2008; 14: 147-56. https://doi.org/ 10.1177/1073858407305850

Chen GF, Xu TH, Yan Y, Zhou YR, Jiang Y, Melcher K, et al. Amyloid beta: structure, biology and structure-based therapeutic development. Acta Pharmacol Sin 2017; 38: 1205-35. https://doi.org/10.1038/aps.2017.28

Cheng J, Shen W, Jin L, Pan J, Zhou Y, Pan G, et al. 
Treadmill exercise promotes neurogenesis and myelin repair via upregulating $\mathrm{Wnt} / \beta$-catenin signaling pathways in the juvenile brain following focal cerebral ischemia/reperfusion. Int J Mol Med 2020; 45: 1447-63. https://doi.org/10.3892/ijmm.2020.4515

Choi $\mathrm{DH}$, Lee $\mathrm{KH}$, Lee J. Effect of exercise-induced neurogenesis on cognitive function deficit in a rat model of vascular dementia. Mol Med Rep 2016; 13: 2981-90. https://doi.org/10.3892/mmr.2016.4891

De Felice FG, Velasco PT, Lambert MP, Viola K, Fernandez SJ, Ferreira ST, et al. Abeta oligomers induce neuronal oxidative stress through an N-methyl$\mathrm{D}$-aspartate receptor-dependent mechanism that is blocked by the Alzheimer drug memantine. J Biol Chem 2007; 282: 11590-601. https://doi.org/10.1074/jbc. M607483200

Doi Y, Takeuchi H, Horiuchi H, Hanyu T, Kawanokuchi J, Jin S, et al. Fingolimod phosphate attenuates oligomeric amyloid $\beta$-Induced neurotoxicity via increased brainderived neurotrophic factor expression in neurons. PLoS One 2013; 8: e61988. https://doi.org/ 10.1371/journal.pone.0061988

Gharebaghi A, Amiri I, Salehi I, Shahidi S, Komaki A, Mehdizadeh $\mathrm{M}$, et al. Treadmill exercise attenuates 3,4methylenedioxymethamphetamine-induced memory impairment through a decrease apoptosis in male rat hippocampus. J Neurosci Res 2017; 95: 2448-55. https://doi.org/10.1002/jnr.24078

Jeon $\mathrm{YK}, \mathrm{Ha} \mathrm{CH}$. The effect of exercise intensity on brain derived neurotrophic factor and memory in adolescents. Environ Health Prev Med 2017; 22: 1-6. https://doi.org/10.1186/s12199-017-0643-6

Kim G, Kim E. The effects of antecedent exercise on motor function recovery and brain-derived neurotrophic factor expression after focal cerebral ischemia in rats. J Phys Ther Sci 2013; 25: 553-6. https://doi.org/10.1589/ jpts.25.5 553

Kim SE, Ko IG, Kim BK, Shin MS, Cho S, Kim CJ, et al. Treadmill exercise prevents aging-induced failure of memory through an increase in neurogenesis and suppression of apoptosis in rat hippocampus. Exp Gerontol 2010; 45: 357-65. https://doi.org/10.1016/ j.exger.2010.02.005

Komaki H, Faraji N, Komaki A, Shahidi S, Etaee F, Raoufi $S$, et al. Investigation of protective effects of coenzyme Q10 on impaired synaptic plasticity in a male rat model of Alzheimer's disease. Brain Res Bull 2019; 147: 1421. https://doi.org/10.1016/j.brainresbull.2019.01.025

Koo JH, Kang EB, Oh YS, Yang DS, Cho JY. Treadmill exercise decreases amyloid $\beta$ burden possibly via activation of SIRT-1 signaling in a mouse model of Alzheimer's disease. Exp Neurol 2017; 288: 142-52. https://doi.org/10.1016/j.expneurol.2016.11.014

Leuner K, Müller WE, Reichert AS. From mitochondrial dysfunction to amyloid beta formation: novel insights into the pathogenesis of Alzheimer's disease. Mol Neurobiol 2012; 46: 186-93. https://doi.org/10.1007/ s12035-012-8307-4

Molteni R, Ying Z, Gómez -Pinilla F. Differential effects of acute and chronic exercise on plasticity-related genes in the rat hippocampus revealed by microarray. Eur $\mathrm{J}$ Neurosci 2002; 16: 1107-16. https://doi.org/10.1046/ j.1460-9568.2002.02158.x

Navarro A, Gomez C, López-Cepero JM, Boveris A. Beneficial effects of moderate exercise on mice aging: survival, behavior, oxidative stress, and mitochondrial electron transfer. Am J Physiol Regul Integr Comp Physiol 2004; 286: R505-11. https://doi.org/10.1152/ ajpregu.00208.2003

Otsuka S, Sakakima H, Sumizono M, Takada S, Terashi T, Yoshida Y. The neuroprotective effects of preconditioning exercise on brain damage and neurotrophic factors after focal brain ischemia in rats. Behav Brain Res 2016; 303: 9-18. https://doi.org/ 10.1016/j.bbr.2016.01.049

Pang PT, Lu B. Regulation of late-phase LTP and long-term memory in normal and aging hippocampus: role of secreted proteins tPA and BDNF. Ageing Res Rev 2004; 3: 407-30. https://doi.org/10.1016/j.arr.2004. 07.002

Patterson SL, Abel T, Deuel TA, Martin KC, Rose JC, Kandel ER. Recombinant BDNF rescues deficits in basal synaptic transmission and hippocampal LTP in BDNF knockout mice. Neuron 1996; 16: 1137-45. https://doi.org/10.1016/S0896-6273(00)80140-3

Paxinos G, Watson C. The rat brain in stereotaxic coordinates: hard cover edition. Academic Press 2006.

Rossi C, Angelucci A, Costantin L, Braschi C, Mazzantini $M$, Babbini $F$, et al. Brain-derived neurotrophic factor (BDNF) is required for the enhancement of hippocampal neurogenesis following environmental enrichment. Eur $\mathrm{J}$ Neurosci 2006; 24: 1850-6. https://doi.org/10.1111/ j.1460-9568.2006.05059.x

Sajadi A, Amiri I, Gharebaghi A, Komaki A, Asadbegi M, Shahidi S, et al. Treadmill exercise alters ecstasyinduced long- term potentiation disruption in the hippocampus of male rats. Metab Brain Dis 2017; 32 : 1603-7. https://doi.org/10.1007/s11011-017-0046-9

Seil FJ, Drake-Baumann R. Neurotrophins and activitydependent inhibitory synaptogenesis. Prog Brain Res 2000; 128: 219-229. https://doi.org/10.1016/S00796123(00)28019-9

Selkoe DJ. Alzheimer's disease: genotypes, phenotypes, and treatments. Science 1997; 275: 630-1. https://doi.org/10.1126/science.275.5300.630

Slipczuk L, Bekinschtein P, Katche C, Cammarota M, Izquierdo I, Medina JH. BDNF activates mTOR to regulate GluR1 expression required for memory formation. PLoS One 2009; 4: e6007. https://doi.org/10.1371/journal.pone.0006007

Sung $\mathrm{YH}$. Effects of treadmill exercise on hippocampal neurogenesis in an MPTP /probenecid-induced Parkinson's disease mouse model. J Phys Ther Sci 2015; 27: 3203-6. https://doi.org/10.1589/jpts.27.3203

Tyler WJ, Pozzo-Miller LD. BDNF enhances quantal neurotransmitter release and increases the number of docked vesicles at the active zones of hippocampal excitatory synapses. J Neurosci 2001; 21: 4249-58. 
https://doi.org/10.1523/JNEUROSCI.21-12-04249.2001

van der Zee J, Sleegers K, Van Broeckhoven C. Invited article: the Alzheimer disease-frontotemporal lobar degeneration spectrum. Neurology 2008; 71: 1191-7. https://doi.org/10.1212/01.wnl.0000327523.52537.86

Vaynman S, Ying Z, Gomez-Pinilla F. Interplay between brain-derived neurotrophic factor and signal transduction modulators in the regulation of the effects of exercise on synaptic-plasticity. Neuroscience 2003; 122: 647-57. https://doi.org/10.1016/j.neuroscience. 2003.08.001

Vicario- Abejón C, Collin C, McKay RD, Segal M. Neurotrophins induce formation of functional excitatory and inhibitory synapses between cultured hippocampal neurons. J Neurosci 1998; 15; 18: 7256-71. https://doi.org/10.1523/JNEUROSCI.18-18-07256.1998 